\title{
Confessione e biografia: per un avvenire fondato nella storia. Note a partire da Jacques Derrida e Jean-Luc Marion
}

\author{
Francesca Peruzzotti \\ (Fondazione san Carlo, Modena - Institut Catholique, Paris, fra.peru@libero.it)
}

\section{Ragioni per un incontro}

Il dialogo tra Jacques Derrida e Jean-Luc Marion relativo alla questione del dono noto, mentre ha ricevuto minore attenzione quello che porta sulla teologia negativa ${ }^{1}$. Eppure, parimenti fecondo confrontarsi con quel plesso di questioni, perch il dibattito istituito consente di approfondire quali forme di singolarità storica consentono di riconoscere il futuro come avvenire possibile, a partire dal rapporto istituito tra la scrittura della teologia negativa e quella biografica.

Le due proposte non sono omogenee, a partire dal comune riconoscimento del ruolo che la forma apofatica ${ }^{2}$ ha avuto all'interno del pensiero occidentale quale alternativa alla metafisica della presenza: per Derrida si tratta di rifiutare un'equivalenza senza resto tra teologia negativa e decostruzione, mentre per Marion la prossimità alla questione teologica decisiva. Entrambi seguono per la medesima linea di sviluppo, l'indagine epistemologica si completa nell'analisi della parola orante della quale enfatizzato il tratto pratico, confessante. La confessione, sviluppata grazie al confronto con l'opera agostiniana e diversamente intesa come auto o etero biografia, sul piano dell'universalità filosofica guadagna una disposizione soggettuale che trova nel rapporto alla storia quale avvenire possibile il nucleo della propria consistenza.

I due Autori mirano a superare il tratto solipsistico del soggetto moderno praticando il campo filosofico che consegue al tramonto della metafisica, il fondamento teorico la regione paradossale che opera con le categorie dell'impossibile, così da favorire l'implicazione personale come necessaria al destino della verità. Il soggetto che confessa s testimoniale, supera il rapporto all'alter e al medesimo secondo forme reciproche in favore di un dramma ternario, ove la distensione temporale riabilita il senso della storia.

\footnotetext{
${ }^{1}$ I due temi sono quantomeno intrecciati, nella misura in cui la questione della teologia negativa sfocia in quella dell'es gibt (cfr. Marion 2012, 123).

2 Gli Autori sono concordi sull'impossibilità di assumere la teologia negativa come un paradigma, questo stile di pensiero si manifesta piuttosto in una pluralità di costruzioni teoriche (cfr. Marion 2001, 157 e Derrida 1999, 43).
} 


\section{Possibilità della filosofia oltre la metafisica: teologia negativa}

Marion ricerca l'adeguata espressione filosofica per una autentica relazione al divino, che non comporti una riduzione idolatrica, ovvero non si limiti alla definizione quale infinita perfezione secondo categorie meramente umane. È necessaria una distanza senza misura tra il soggetto finito e il Dio cui costui si rivolge perché il primo sia libero e Dio non cada in una antropologizzazione. Il divino della onto-teologia è squalificato, è piuttosto il Dio della rivelazione cristiana a favorire una relazione evitando la piena trasparenza che annullerebbe i termini in gioco. $\mathrm{DXo}^{3}$ non è limitato dalla concettualità cui neppure la definizione secondo l'eminenza d'Essere si sottrae. Parimenti la libertà antropologica è recuperata grazie al discorso di lode, che non è informativo né crea un idolo, ma lascia essere il divino e convoca l'uomo ad uno sviluppo personale.

Per offrire un adeguato apparato concettuale che esprima secondo l'universalità filosofica quanto è anticipato dal pensiero del cristianesimo ${ }^{4}$ Marion si rivolge al dispositivo della differenza ontologica formulato da Martin Heidegger, escluso in quanto ancora implicato ontologicamente e, in seconda battuta, alla différance derridiana. Essa parrebbe confermare la ricerca, poiché la traccia che la caratterizza è segno di un'assenza impossibile da ascrivere al registro della semplice presenza, lasciando permanere solo l'indicazione della sua cancellazione. La différance radicalizza «l'indifferenza della differenza» (Marion 1977, 227), tuttavia lo sviluppo per contrasto rispetto alla differenza ontologica svela la sua aporia: avocando a sé l'essere originaria, riproduce l'apparato metafisico che intendeva oltrepassare. Entrambe non sono risolutive, la nominazione divina rimane idolatrica o perché costretta nel rapporto ontico/ontologico o perché comporta l'impossibilità di riferirsi a Dio, con la squalifica irrimediabile di qualsiasi pensiero teologico, rovesciamento speculare dell'aporia iniziale, non ancora suo superamento ${ }^{5}$. Marion fa

3 Il Dio crociato permette il duplice riferimento alla rivelazione teologica nella croce del Figlio e alla barratura dell'Essere proposta da Heidegger quale indice del superamento dell'epoca metafisica.

${ }^{4}$ La filosofia marioniana si sviluppa anche nel confronto con alcune figure della teologia novecentesca. Su tutti, Hans Urs von Balthasar per ragioni biografiche e speculative segna la teoria dell'Autore (cfr. Marion 2012a, 45-54). Inoltre è da considerare la formazione culturale e religiosa che ha connotato gli universitari cattolici parigini grazie all'impulso di Maxime Charles prima e di Jean-Marie Lustiger poi (cfr. Lemna 2016, 248-249). Una risonanza di tale spiritualità, fondata sull'adorazione eucaristica, può essere trovata nelle analisi relative alla teologia negativa.

${ }^{5}$ La differenza che intercorre tra Marion e Derrida può essere meglio illustrata una volta rintracciato l'epistolario paolino tra le fonti dell'alternativa speculativa tra idolo e icona, così da rinvenire una prima formulazione dell'intreccio tra visibile ed invisibile nella singolarità concreta di Cristo, immagine del Dio invisibile (Col 1,15) (cfr. Tarditi 2015, 154 ss.). Nel cristianesimo da cui Marion prende le mosse l'invisibile è fondato da una manifestazione storica, mentre l'ebraismo che segna Derrida è connotato da un'attesa infinita, fino alla formulazione paradossale di un'attesa messianica senza Messia (cfr. Tarditi 2008, 154). 
coincidere la centralità della différance con la chiusura dell'orizzonte teorico della decostruzione, incapace di ipotizzare un dio ulteriore a quello ontoteologico ${ }^{6}$, escludente la teologia negativa dalle forme di pensiero significative, quindi risposta non conveniente al suo stesso pungolo teoretico ${ }^{7}$.

Stante la radicalità del dialogo ogni ulteriore sviluppo parrebbe impedito, al contrario, proprio il concetto di Dio e le prossimità tra différance e teologia negativa costringono Derrida ad un approfondimento ulteriore, che avrà tra le sue conseguenze proprio l'attribuzione di un credito non previsto alla proposta marioniana (Derrida 1987b, 211, nota 18 e Derrida 1994, 155).

L'ingresso di Derrida nel dibattito puntualizza la portata della teologia negativa, da pratica di linguaggio a esperienza di pensiero. Rifiutando l'identificazione tra la decostruzione e il dire apofatico non scredita il secondo ${ }^{8}$, precisando altresì che è necessario perfezionare entrambi i termini ${ }^{9}$. La pretesa di accostarsi a Dio anteponendo a qualsivoglia determinazione la semplice negazione è speculativamente ingenua, in quanto ripete un concetto, pur rovesciato; con un discorso carente di ragioni si maschera l'incapacità di trovarne. L'Autore rintraccia in ogni sistema formulato, sia esso esplicito, come quello di Dionigi o di Eckhart, o determinante sul piano teorico, come quello heideggeriano, un «rialzo ontologico dell'ipersostanzialità» (Derrida 1987b, 178), che non basta però a squalificare irrimediabilmente la teologia negativa, propone infatti un contributo onde mantenere l'apporto costruttivo offerto alla teoresi.

L'ambito autobiografico fornisce la giustificazione fondante l'approccio: non è trascurabile che Denegazioni sia pronunciato a Gerusalemme, né che la redazione di Salvo il nome avvenga a Nizza, in occasione dell'agonia materna ${ }^{10}$. Tematizzare la teologia negativa è possibile a prezzo del suo esercizio, la cui pratica avviene solo in regime confessante. La confessione stabilisce l'apporto adeguato della prima persona allo svolgersi del pensiero: non più soggetto produttore di teoria, ma scrivente che si scopre legato ad una precedenza che lo trascende ${ }^{11}$.

\footnotetext{
${ }^{6}$ L'esclusione è netta già in Derrida 1972, 33.

7 Marion denuncia indebita l'equivalenza posta nel saggio La différance tra metafisica, onto-teologia e teologia, essa sarebbe stabilita al solo scopo di rimarcare l'originalità della categoria derridiana, che al contrario troverebbe proprio nella teologia negativa l'insidia alla sua novità (cfr. Marion 1977, 253, nota 40).

8 «Si è detto affrettatamente che [la decostruzione] era una specie di teologia negativa (non è ne vero né falso)» (Derrida 1987a, 10).

${ }^{9}$ Sia Derrida 1987b che Derrida 1987a, la cui prima stesura appartiene a periodi diversi, fanno uso delle medesime parole per approssimare decostruzione e teologia negativa.

10 La biografia di Derrida, per ciò che concerne il suo rapporto all'ebraismo, è significativa per comprendere il ruolo della teologia negativa. Infatti la tradizione biblica, a partire da Es 3,14, restituisce l'impossibilità di rapportarsi a Dio limitandosi ad una definizione che prescinda da una storia condivisa (cfr. Geraci 2010, 139146.150-156).

${ }^{11} \mathrm{La}$ confessione è originariamente unita alla circoncisione: Circonfessions, l'autobiografia che Derrida fornisce di sé, è scrittura ospitata in un altro testo, non è la
} 
Si scrive di sé a causa dell'altro e in vista di lui, particolarmente quando incombe il pericolo di perderlo e il legame si dà nella forma dell'assenza ${ }^{12}$. Chi non è presente si manifesta nella traccia paradossale della promessa, che rassicura circa la parzialità della sua sottrazione. Sia la teologia negativa che la promessa sono atti di parola, la loro equivalenza decide il legame tra gli uomini, la teologia negativa «è legata forse a una promessa, quella dell'altro, che io debbo mantenere perché mi impegna a parlare là dove la negatività dovrebbe condurre il discorso alla sua assoluta rarefazione» (Derrida 1987b, 184).

L'assenza di contenuto riconducibile al dominio informativo accomuna l'interlocutore al locutore attorno ad un senso sperato, se si dà una promessa è impossibile che questa termini nel nulla assoluto. Seguendo il medesimo schema il discorso apofatico dona il riferimento alla trascendenza purché si riconosca inammissibile il ricorso ai canoni del dire predicativo. Occorre trovare lo stile conveniente, capace di sostare nell'impossibilità radicale che, proprio dicendo, afferma che dire non si può.

L'oltrepassamento delle logiche metafisiche comporta un approccio alternativo alla nominazione. Generalmente, tramite la limitazione e la distinzione, il nome consente di individuare. La decostruzione, al contrario, opera eccettuando il nome, perché ne sia preservata ogni potenzialità, sia cioè salvato ${ }^{13}$. Derrida generalizza quanto la sapienza biblica suggerisce a proposito dell'impossibilità di pronunciare il tetragramma divino, perché se venisse nominato sarebbe operata una contestuale riduzione tanto della divinità che dell'umanità; globalmente, ogni tentativo di esporsi all'altro deve prescindere dal definirlo, andare oltre lui onde preservarne la trascendenza irriducibile.

Le varie forme di teologia negativa teorizzate lungo la storia della metafisica sono quindi squalificate, Derrida ritiene che anche la via dell'eminenza non si sottragga alla sottile dinamica della nominazione, piuttosto nasconda sostanzialità. La soluzione è racchiusa nell'esclusione di qualsiasi filosofia pertinente alla divinità, infatti la decostruzione va oltre, riferendosi al totalmente altro in chiave universale (cfr. Derrida 1967). Il linguaggio si rinnova rinunciando alla funzione predicativa ${ }^{14}$ e trovando lo stile che gli è adeguato nella confessione, che stravolge i canoni linguistici (cfr. Derrida 1987b, 182); la teologia negativa è correttamente intesa se riproduce la stessa dinamica, forzando il dicibile (cfr. Derrida 1994, 160), per questo motivo specificare lo statuto dell'alterità non è l'obiettivo primario: «la struttura della traccia è in generale la possibilità stessa di un'esperienza della finitudine, la distinzione tra

pretesa dell'ego sovrabbondante di eccedere ogni limite storico, ma la possibilità di dire sé a partire da limiti e cesure che consentono la vita (cfr. Lettieri 2001,544).

12 La consapevolezza dell'approssimarsi della propria morte è favorita solo dalla morte dell'altro, esemplare Derrida 1991 (cfr. Ferraris 2008, 115).

13 «Perdere il nome, questo non è perdersi in lui, distruggerlo o ferirlo. Al contrario, è semplicemente rispettarlo: come nome» (Derrida 1994, 152).

${ }^{14}$ La mutazione avviene considerando il linguaggio orante la forma adeguata per il nuovo paradigma. 
una causa finita e una causa infinita della traccia pare qui, oseremmo dire, secondaria. È essa stessa un effetto di traccia o di différance» (Derrida 1987b, 199).

\section{Dare forma alla parola: preghiera e nominazione}

Marion si pone in dialogo con il postmoderno accogliendo l'impossibilità, radicale dopo Heidegger, di riferirsi a Dio secondo le categorie della metafisica, tuttavia non abbandona irrevocabilmente il concetto ${ }^{15}$ : è stato cruciale lungo l'intera storia della filosofia e rifiutarlo equivarrebbe ad una cieca limitazione della riflessione ${ }^{16}$. Proprio la considerazione di questa regione paradossale consente di superare le frontiere che ricondurrebbero la filosofia a mera descrizione di ciò che si può conoscere senza incremento del sapere. Si fa allora necessario individuare quale sia la forma adeguata per riferirsi a ciò è refrattario ad ogni classificazione, in quanto ad essa eccedente, ovvero il paradosso (cfr. Marion 2010, 277-284).

Marion rimprovera a Derrida l'identificazione del procedimento per eminenza alla dinamica metafisica, così da impedire approcci ulteriori. Egli tratteggia piuttosto una terza via non riducibile alla definizione secondo la via positiva, catafatica, e quella negativa, data dalla negazione di ogni attributo. Essa è la più autentica, fonda senza cancellare le altre due. Qualunque nominazione, definendo il soggetto, ne riduce il profilo originariamente eccessivo, parimenti, la rinuncia che opta per il mutismo recide il legame tra l'atto che implica il soggetto e la verità. La terza via, invece, non segue le strade del concetto, perché è pragmatica: «non solo non si tratta più di dire (o negare) qualcosa di qualcos'altro, ma di non dire più, né dedurre (de-dire) - si tratta di riferirsi a Colui che non è più toccato dalla nominazione, non più dire il referente, ma riferire pragmaticamente il locutore al Referente inaccessibile» (Marion 2001, 171). La de-nominazione supera la nominazione perché conserva insieme la necessità di nominare e di cancellare il processo di designazione, quando ogni intento predicativo è interrotto il soggetto deve rivolgersi al divino in altro modo, più consono alla sua alterità.

Neppure la referenza attraverso un nome proprio è risolutiva, perché indirizzabile ad una pluralità di soggetti: l'attribuzione di nomi e aggettivi è sempre inadeguata rispetto al referente cui sono rivolti, il nome proprio non

\footnotetext{
15 Marion specifica che vi è un approccio onto-teologico qualora tre elementi siano presenti contemporaneamente: la formalizzazione del concetto di ente, il suo uso per definire univocamente Dio e le creature, il legame tra questi secondo la figura del principio o della causa, L'Autore ritiene non sia questo il caso né con Dionigi, né con Tommaso (cfr. Marion 2001, 175 e Marion 2002, 11-13, 241-286).

${ }^{16}$ L'approfondimento degli aspetti speculativi legati alla teologia negativa può favorire un differente approccio alla storia della metafisica, che rivaluti l'apporto di figure, come quella di Descartes, altrimenti considerate in modo univoco (cfr. Gschwandtner 2016, 20-24).
} 
individua, ma universalizza, pertanto mette in luce il linguaggio adeguato agli umani che non limita né perde il legame con il soggetto. La base metodologica è consentita dalla fenomenologia, che riconfigura la via per eminenza del pensiero apofatico, non più relegata ad un sostrato biblico o religioso. Il fenomeno saturo, la cui datità eccedente non sarà mai significabile da una coscienza ad esso correlata, consente di comprendere il divino filosoficamente e diventa paradigma per autentica fenomenalità, che trova nell'amore la sua universalizzazione (cfr. Marion 2001, 193).

La soggettività non è annichilita, nuova è però la figura secondo la quale si correla al dato in sovrappiù, non ha ormai alcuna pretesa su di esso, solo si rivela incessantemente convocata. La teologia negativa si rinnova alla luce della relazione tra fenomeno saturo e adonato, poiché delinea una legalità secondo la quale nel riferirsi a Dio non vi è alcuna antecedenza o a-priori concettuale, ma la possibilità di rispondere in seconda battuta, pragmaticamente.

Indagando la fenomenalità erotica si guadagna la globalità della saturazione, la sua banalità piuttosto che la rarità; di particolare interesse la ridefinizione del linguaggio portata dall'amore, perché il legame vicendevole è espresso in molte forme linguistiche, ma è estraneo a qualsiasi corollario nozionistico. Nel contesto amoroso la parola non opera definendo, ma lega tra loro gli interlocutori, presenti o assenti. La manifestazione vocale dell'amore culmina quando l'amante non enuncia alcunché, la massima espressione si dà quando manca contenuto predicativo sia nei confronti del referente dell'amore, che di coloro che vi assistono (cfr. Marion 2005, 114). L'Autore puntualizza che «non si tratta di affermare o negare qualcosa su qualcosa, ma si tratta di agire sull'altro e lasciarlo agire su di me» (Marion 2005, 126).

La dimensione erotica non esclude che alla persona amata siano attribuiti nomi (cfr. Marion 2003, § 28), essi sono anzi sovrabbondanti, ma nessuno potrà approssimare uno stato di cose, la copiosità è la figura paradossale che il nome porta inscritta, secondo la de-nominazione.

L'omofonia consentita dalla lingua francese tra nome e negazione (nom-non) guida Marion verso la risoluzione della questione: il riferimento al divino o all'amato avviene in regime pragmatico perché la lode non intende descrivere, ma manifestare un vincolo decisivo. Chi persiste nella nominazione verifica un rovesciamento, delinea cioè la propria parzialità insieme alla nullità del nome impiegato rispetto alla realtà cui si relaziona.

Il guadagno ricade sulla finitezza, il soggetto si riconosce convocato a ricevere identità, come sinteticamente avviene nei segni battesimali, in cui «lungi dall'attribuire a Dio un nome intelligibile per noi, entriamo nel suo Nome impronunciabile, come ricevendone in sovrappiù il nostro» (Marion 2001, 189). Il soggetto è adonato perché riceve da altri la propria identità, il nome corrisponde all'appellativo per essere sé. Non è una determinazione, ma l'evento che dona un'identità nuova, oltre l'effettuale, da viversi nella distensione storica. 
Per la consistenza del soggetto, adonato, è necessario un debito originario, che il semplice nome porta alla luce mostrando il tratto aporetico, la finitezza si trascende proprio insistendo sul finito. Il nome che de-nega, legando la nominazione alla sua falsificazione, non definisce nulla di colui al quale si riferisce, ma fa ricadere il risultato su ciò che gli è correlato, convocando un soggetto e nominando l'umano.

Entrambi gli Autori concorrono a delineare la forma di pensiero adeguata per riferirsi all'impossibile, rovesciamento che coinvolge l'espressione della soggettività e di conseguenza il suo tratto finito e storico; essi focalizzano nella preghiera la forma linguistica che supera le aporie del linguaggio predicativo. Rivolgendosi alla divinità ma evitando di definirla si ottiene uno spazio in cui il soggetto è responsabile di sé e per Dio, senza scadere nell'estenuazione apofatica della mancata individuazione. La preghiera offre «la promessa della sua [dell'altro] presenza come altro, e in fondo la trascendenza della sua stessa alterità, senza alcuna altra determinazione» (Derrida 1987b, 211).

La trascendenza che è in gioco nella preghiera è valutata diversamente da Marion e Derrida, per il primo si tratta ancora di quella divina, per il secondo questa è inessenziale, la trascendenza mette in moto la questione permanendo inconoscibile, anzi evitando di determinare la sua esistenza reale. Con Marion è ancora necessario uno statuto personale che fa di Dio l'irriducibile, ma entrambe le posizioni consentono di svelare l'alterità nel cuore dell'ipseità, di essa costitutiva.

Chi loda pronuncia parole che lo inseriscono nell'ascolto di ciò che lo precede (cfr. Marion 2001, 178-179). Pregando il soggetto percepisce il proprio ritardo, si ripropone la distanza necessaria affinché la prima persona abbia consistenza e nel contempo non sviluppi pretese sull'origine. Con il legame si mantiene la validità di entrambi i poli: non è reciproca data la loro incommensurabilità, ma un debito che equilibra entrambe le libertà. La prima è ravvisabile nell'eccedenza che genera oltre sé, la seconda nella necessità della sua affermazione affinché l'origine sia visibile.

La preghiera è rito, l'atto di parola è estraneo alla solitudine, avviene secondo lo schema liturgico, corale, ove è ancor più evidente la funzione performativa (cfr. Marion 1996, 114): non un suono di parole, ma il gesto che, implicando la sospensione di qualsiasi fare produttivo, rintraccia l'essenza dell'umano. La preghiera, momento di intima consapevolezza, evita il solipsismo e l'autoinganno, non mima la forma dialogica, piuttosto è possibile grazie ad una triangolazione, quella consentita dalla comunità di coloro che insieme si riferiscono all'origine. Pertanto fa nascere anche la determinazione singolare, dalla struttura patica è consentita la drammatica propria di un legame personale. Infatti, modello di ogni preghiera è ravvisato nella confessione, forma che consente di esprimere la storicità della propria singolarità insieme alla sua dimensione costantemente plurale. 
La forma più raffinata di solipsismo, che pone la soggettività in confronto unicamente con la sua origine, non è risolutiva, mentre parlare di Dio è possibile purché vi sia un dialogo (cfr. Derrida 1994,129), messo in luce dalla confessione scritta dopo l'esperienza. Riferendosi alla storia passata, pertanto, si delineano le tracce per darne una diversa interpretazione futura, affidandole ad altri.

Tanto nel regime erotico che in quello teologico la conclusione della relazione dinamica si apre ad una storia, nella quale è l'altro uomo ad essere implicato; come sintetizza la narrazione evangelica del dialogo post pasquale tra Pietro e Gesù $(G v 21,17)$ si tratta di un terzo, solo obbedendo al comando di andare verso i fratelli si attua l'amore verso Cristo altrimenti messo in dubbio (cfr. Marion 2005, 128-129).

\section{La confessione: de-misura umana nella storia}

La storicità dell'attuazione singolare si fonda sugli aspetti centrali della teoria derridiana. La scrittura è compresa come traccia, seguendo i due significati che la différance racchiude: essere in ritardo, secondo una distensione temporale, differire, non riproponendo mai il medesimo. La parola scritta è il medio che distanzia dal medesimo, facendo sì che anche la determinazione di sé sia esperienza di alterità. La forma confessante è sempre posteriore rispetto ad un evento concluso, se già il primo ha la forma del documento, solo il secondo, scritturistico, lo manifesta pienamente, è necessario un post-scriptum per compiere l'evento. Esemplari le Confessioni agostiniane: non sono redatte per aumentare la conoscenza del vissuto del vescovo di Ippona né nei confronti di sé medesimo, che già conosce quanto avvenuto, né verso Dio, onnisciente per definizione; ugualmente necessaria, però, la loro scrittura, perché in quella storia personalissima altri sono implicati con uguale importanza. Le Confessioni, infatti, sono scritte per i fratelli, in virtù della carità che muove nei loro confronti, perché essi determinino di conseguenza la loro esistenza.

La scrittura trova nella testimonianza l'alternativa al rispecchiamento dialogico, dove si smarrisce l'individualità dei soggetti in gioco. Chi testimonia non riproduce sé, ma l'altro che ha preceduto, ponendolo per un terzo senza duplicarsi, piuttosto implicando sé stesso; la parola si accompagna all'atto, essendo atto essa stessa. Colui al quale la testimonianza è rivolta non potrà fare altro se non agire a propria volta perché la testimonianza è offerta autenticamente se intrecciata alla determinazione personale. La dinamica è compiuta nella scrittura perché, seguendo Derrida, essa lascia imprendibile l'origine dalla quale si dispiega e chiama in causa colui al quale si rivolge ${ }^{17}$. Il tratto ermeneutico è essenziale, pone in essere la differenza, permette alla verità multiforme di avere nuova attuazione ${ }^{18}$. Parimenti, essa rimane valida

17 Sulla dimensione testimoniale della scrittura, cfr. Dalmasso 1994, 21.

18 «La confessione (come la scrittura che la espone) è metafora della metafora, differimento mai appropriato della verità, infinito ritrarsi del proprio nell'invocazione, 
solo se ha una referenza ulteriore. Se, come nelle Confessioni, l'esperienza personalissima è riconosciuta attestazione di un'origine, promettente e per questo dicibile, allora non vi è nullificazione. Nella teologia negativa il nome di Dio attesta la medesima dinamica, esso è pari ad una freccia che senza interruzione indica la direzione da percorrere. Non identifica, né trattiene la ricerca, ma propone un agire e un'acquisizione di responsabilità 19 .

Anche per Marion l'apporto di Agostino è esemplare, il confronto diretto con il suo pensiero consente all'Autore una sintesi delle innovazioni proposte nell'alveo fenomenologico e porta a compimento il senso della sua opera. Il discorso di lode si sviluppa nella confessione, «forma originale di ogni possibile parola» (Marion 2008, 65), atto ove è primario l'intreccio con il campo dell'esperienza, definendo una triangolazione tra il soggetto, altri, Dio. La lode arriva al postulato preservando una distanza infinita rispetto al postulante, così da dare spazio alla totalità del mondo e la confessione, seguendo la stessa dinamica, implica i fratelli nel riferimento a Dio. Ancor più della lode questa evita di definire il suo referente, ma conserva con esso un legame fondamentale, che coinvolge la vita del singolo consegnata a Dio.

La confessione si avvicina al dominio dell'autobiografia per rovesciarlo: la sua forma autentica non è quella moderna (Michel de Montaigne, Jean-Jacques Rousseau), soggetta ad una riduzione pari a quella del cogito cartesiano, solipsista; lo scritto di Agostino è archetipo della etero biografia, in cui il soggetto, eccentrico a sé medesimo, rinviene la propria autenticità parlando di sé perché si scopre già detto da Dio, la dimensione intima è legata a quella pubblica (Marion 2008, 68). L'andamento ternario evita il rispecchiamento orizzontale, Dio si manifesta nella forma dell'assente ${ }^{20}$ e apre lo spazio della storia, un avvenire non preventivabile ma assumibile nella forma della risposta all'evento. La confessione è l'effettuazione pragmatica della preghiera: l'intreccio tra la parola e l'azione non si risolve nel riconoscere la parola agita

invio senza destinatario assicurato: [...] quasi che, nella confessione, Dio venisse chiamato a venire nell'esistenza, ad esistere nel differire, quasi che Dio fosse il nome dell'a-venire stesso che costituisce la singolarità» (Lettieri 2014, 158-159).

${ }^{19}$ L'immagine della freccia proposta in Derrida $(1994,154-155)$ richiama quella della testimonianza, il Battista, testimone per eccellenza, si risolve nell'atto di indicare oltre sé, portando a Cristo.

20 «Come terzo, Dio si trova non solo ridotto a qualcosa su cui sto per dire qualcosa, ma soprattutto ridotto a quell'ille che sto per escludere, o meglio che sta per escludersi da sé dallo spazio dialogico, prendendo lo statuto di un semplice terzo escluso, di un oggetto in assenza del quale ipse (io) e iste (tu, questo altro me) stiamo per parlarci parlando su di lui» (Marion 2008, 31). L'assenza è già tratto fondante dell'autenticità erotica, possibilità che questa non appartenga al dominio ontologico (cfr. Marion 2005, 115). 
esclusivamente nella liturgia, ma nel fatto che questa viene dalla vita e ad essa conduce, lega il passato e il futuro in un presente costitutivo dell'avvenire ${ }^{21}$.

Il medio testuale è fondamentale per fissare la biografia come originariamente alterata, la scrittura della propria confessione, storia tra l'io e Dio, è immantinente efficace per altri, altrimenti sarebbe vana pretesa di gloria auto attribuita, «le Confessiones si dispiegano come un testo di lode, una lode divenuta testo, più esattamente come un testo che si offre alla lettura come è stato scritto» (Marion 2008, 32).

L'agire confessante comporta l'attuazione dell'intreccio tra appello e risposta tipico della fenomenologia della donazione. La continuità dell'appello è assicurata, la risposta media verso altri la chiamata ricevuta, «la confessio non si riduce quindi a un gioco linguistico o a un atto di parola tra le altre, ma definisce l'uso di ogni parola, quando devo dire me stesso in quanto tale, cioè davanti a Dio» (Marion 2008, 52). Una confessione può realizzarsi solo se agita e raccontata, la forma narrativa è necessaria perché il passato raccolto nella memoria si trasformi nell'avvenire immaginato. Si conferma la coappartenenza di erotico e teologico, in entrambi i dominî il soggetto confessante non si limita ad un raccordo tra l'atto e il suo racconto, ma «tale atto parla in quanto interpella» (Marion 2005, 122): chiamando in causa il partner ed attendendo la sua risposta apre alla storia, possibile perché intrecciata in un permanente scambio delle posizioni tra chi chiama e chi risponde. Entrambi sono ugualmente fondati dalla chiamata originaria e mai visibile di Dio, lo spazio che il sé scopre a lui interno è favorito dall'alterità somma, la storia è possibile perché racchiusa in quella Sacra, che si è resa narrabile in parole umane facendosi vita 22 .

I soggetti in relazione sono abilitati a riconoscere il senso pieno della loro scelta perché il terzo testimoniale è tanto originario quanto escatologico (cfr. Marion 2005, 274-283), la sua presenza comporta l'impossibilità di una fuga verticale, la costruzione di un avvenire permane nel riconoscimento del tratto evenemenziale che caratterizza la storia, come si precisa nell'intreccio tra le rispettive scritture e letture ${ }^{23}$. Conseguentemente non vi è un piatto rispecchiamento, ma l'accrescimento iperbolico già conosciuto nella via eminente della teologia negativa, «se soltanto l'istante successivo potrà dare una

${ }^{21}$ L'avvenire si intende alternativo al futuro, quest'ultimo è prosecuzione cronologica del dispositivo metafisico presentificante, mentre il primo corrisponde alla dimensione escatologica che caratterizza già la storicità (cfr. Marion 2008, 249-299).

22 «Le Scritture non consegnano dunque una parola morta di Dio su se stesso, ma offrono la parola silenziosa che, dapprima (da quel prima immemoriale di una creazione), rende possibile la mia propria parola vivente» (Marion 2008, 37).

23 «Come tra l'autore e Dio, così anche tra l'autore e i lettori vengono a porsi una domanda e una risposta: ma con una differenza essenziale: se la domanda (centrifuga) proviene evidentemente dall'autore che confessa ai lettori, la risposta (centripeta) non li rimanda più all'autore, ma rimanda ognuno di essi direttamente a Dio» (Marion 2008, 64). 
qualche risposta a quanto ho chiesto nell'istante precedente, lo scarto temporale tra il dubbio e la conferma non sarà mai colmato ma si prolungherà di domanda in risposta, senza fine. Così, anche nel caso di una risposta positiva, il differimento e la distanza temporale manterranno sempre una différance» (Marion 2008, 124).

\section{Conclusioni}

La confessione è dimensione originaria per i soggetti che trovano nella storia la propria consistenza. Essa, più generalmente, caratterizza la qualità della filosofia capace di operare a seguito della fine dell'epoca metafisica: la stessa filosofia è chiamata a collocarsi con sapienza all'interno della sua storia, così da non convertirsi in mera storiografia ed insieme prospettando un ruolo che eviti una riduzione aprioristica della complessità.

Il breve percorso esposto è eloquente in merito ai fecondi intrecci che nel postmoderno danno nuova vita ad autori e tematiche classici per il pensiero filosofico. Innanzitutto, lo stile adottato dai due Autori istruisce circa il parallelismo che intercorre tra la descrizione delle forme soggettuali e le scelte epistemologiche adeguate all'epoca. Le indagini della teologia negativa non possono essere confinate in un passato ormai privo di incidenza sull'accadere presente, ma restano repertorio che interpella circa le frontiere e le soglie intercettate dall'interrogazione filosofica, sempre spinta ad infrangerle.

Tanto Marion quanto Derrida hanno rinvenuto nell'impossibile, in ciò che si sottrae alla presentificazione della previdibilità, la cifra in grado di aprire nuovi percorsi: l'accadere non coincide con il mero effettuabile, nè il futuro con il calcolo causale. Le possibilità di un pensiero dell'avvenire, rigoroso e privo delle tutele fornite dalla metafisica della presenza, delineano quindi tracce che subito sono significative anche per la comprensione del presente. Esso è ben più dell'attimo puntuale perchè reso tempo disteso dalla promessa di un futuro mai completamente esauribile.

Il sè, non limitato dall'alterazione posta dall'altro e dalla storia, si manifesta in una narrazione che emerge rispetto alla catena cronologica e porta in evidenza il ruolo impreteribile che lo singolarizza. L'eccedenza della storia e l'impossibilità di una sua totalizzazione è consegnata alla scrittura, la sua traccia è manifestazione delle assenze che la costituiscono. Pertanto, l'appello universale che la scrittura mostra rispondendogli riproduce in forma simbolica la sua destinazione.

\section{Bibliografia}

Caputo, J. D. 1997. Prayers and Tears of Jacques Derrida. Bloomington Indianapolis: Indiana University Press.

Dalmasso, G. 1994. Dare il nome. In J. Derrida, Il segreto del nome. Milano: Jaca Book, 7-34. 
Derrida, J. 1967. Violence et métaphysique. In Id. L'écriture et la différence. Paris: Seuil; tr. it. 1971. It. Violenza e metafisica. In Id. La scrittura e la differenza. Torino: Einaudi, 149-169.

1972. La différance. In Id. Marges - de la philosophie. Paris: Minuit; tr. it. 1997. Margini. Della filosofia. Torino: Einaudi, 27-57.

1987a. Lettre à un ami japonais, in Id. Psyché. Inventions de l'autre II. Paris: Galilée; tr. it. 2009. Lettera a un amico giapponese. In Id. Psyché, vol. II. Milano: Jaca Book, 7-13.

1987b. Comment ne pas parler. Dénégations. In Id. Psyché. Inventions de l'autre II. Paris: Galilée; tr. it. 2009. Come non parlare. Denegazioni. In Id. Psyché II. Milano: Jaca Book, 171-236.

1991. Circonfessions. In Id. \& G. Bennington, Jacques Derrida. Paris: Seuil; tr. it. 2008. Derridabase. Circonfessione. Roma: Lithos.

1994. Saufle nom. Post-scriptum. Paris: Galilée; tr. it. 2005. Salvo il nome. Post-scriptum. In Id. Il segreto del nome. Milano: Jaca Book, 129-177. 1999. Derrida's Response to Jean-Luc Marion. In J. D. Caputo \& M. J. Scanalon (ed.) God, the Gift and Postmodernism. Bloomington Indianapolis: Indiana University Press, 42-45.

2005. Composing "Circumfession". In J. D. Caputo \& M. J. Scanalon (ed.) Agustine and Postemodernism: Confessions and Circumfession. Bloomington and Indianapolis: Indiana University Press, 19-27.

Ferraris, M. 2008. Introduzione a Derrida. Roma-Bari: Laterza.

Geraci, S. 2010. L'ultimo degli ebrei. Jacques Derrida e l'eredità di Abramo. Milano-Udine: Mimesis.

Gschwandtner, C. M. 2016. Marion and Theology. London - Oxford - New York - New Delhi - Sidney: Bloomsbury.

Lemna, K. 2016. Jean-Luc Marion and the Theological "School" of Montmartre. Irish Theological Quarterly, 81, 246-266.

Lettieri, G. 2001. L'altro Agostino. Ermeneutica e retorica della grazia dalla crisi alla metamorfosi del De doctrina christiana. Brescia: Morcelliana. 2014. Il differire della metafora, II. Filosofia e Teologia, 28, 127-171.

Marion, J.-L. 1977. L'idole et la distance. Paris: Grasset; tr. it. 1979. L'idolo e la distanza. Milano: Jaca Book. 1996. La croisée du visible. Paris: PUF. 1997. Etant donné. Essai d'une phénoménologie de la donation. Paris: PUF; tr. it. 2001. Dato che. Saggio per una fenomenologia della donazione. Torino: SEI. 2001. Au nom ou comment le taire. In Id. De surcroît. Paris: PUF, 155195.

2002 (1982). Dieu sans l'être. Paris: PUF; tr. it. 2008. Dio senza essere. Milano: Jaca Book.

2003. Le phénomène érotique. Six méditations. Paris: Grasset; tr. it. 2007. Il fenomeno erotico. Sei meditazioni. Siena: Cantagalli. 
2005. Ce qui ne se dit pas - l'apophase du discours amoureux. In Id. Le visible et le révélé. Paris: Cerf; tr. it. 2007. Ciò che non si dice: l'apofasi del discorso amoroso. In Id. Il visibile e il rivelato. Milano: Jaca Book, 107129.

2008. Au lieu de soi. L'approche de Saint Augustin. Paris: PUF; tr. it. 2014. Sant'Agostino. In luogo di sé. Milano: Jaca Book.

2010. Certitudes négatives. Paris: Grasset; tr. it. 2014. Certezze negative. Firenze: Le Lettere.

2012a. La rigueur des choses. Entretiens avec Dan Arbib. Paris: Flammarion.

2012b. Jacques Derrida et l'impossibilité du don. In Id. Figures de phénoménologie. Husserl, Heidegger, Levinas, Henry, Derrida. Paris: Vrin, 117-128.

Tarditi, C. 2008. Con e oltre la fenomenologia storica. Le eresie fenomenologiche di Jacques Derrida e Jean-Luc Marion. il Nuovo Melangolo: Genova. 2015. Seeing the Invisible. Jean-Luc Marion's Path from Husserl to Saint Paul. In A. Cimino \& P. Kontos (ed.) Phenomenology and the Metaphysics of Sight. Leiden-Boston: Brill, 142-162. 
Francesca Peruzzotti

(Fondazione san Carlo, Modena - Institut Catholique, Paris, fra.peru@libero.it)

Confessione e biografia: per un avvenire fondato nella storia.

Note a partire da Jacques Derrida e Jean-Luc Marion

\begin{abstract}
Il testo si propone di approfondire il confronto tra Jacques Derrida e Jean-Luc Marion circa il peso speculativo da attribuire alla teologia negativa. La ricostruzione del incontro mette in luce significativi apporti in ordine alla ridefinizione del soggetto in ambito post metafisico, considerando le possibilita ancora aperte per una storicita non predeterminata a partire dalla presenza. Il futuro non e totalizzazione della storia secondo la fine, ne mera continuazione del presente, bensı evento escatologico, ove il rapporto con l'altro e determinante per la costituzione del se. La figura sintetica che esprime tale intreccio e quella confessante, ove il legame tra passato e futuro non e causalmente determinato, ma implicazione singolare e testimoniale, come illustrato dal capolavoro agostiniano, riferimento irrinunciabile per entrambi gli Autori.
\end{abstract}

This paper aims to draw a connection between Jacques Derrida and JeanLuc Marion in regard to the role of negative theology. This scrutiny shows meaningful contributions of the Authors to a new definition of subjectivity in a post-metaphysical age, and their consideration about which possibilities are still open for a non-predetermined history given outside of the presence domain. The future is neither a totalisation of history by its end, nor a simple continuation of the present. It is an eschatological event, where the relationship with the other plays a crucial role for the self-constitution. Such an interlacement is generated by the confession, where the link between past and future is not causally determined, but instead it is self-witness, as in Augustine's masterpiece, essential reference for both the Authors.

Keywords: Jacques Derrida, Jean-Luc Marion, (Auto)Biography, Confession, Eschatology, History, Negative Theology, Prayer.

Ethics in Progress (ISSN 2084-9257). Vol. 8 (2017). No. 1, Art. \#4, pp. 61-74.

Creative Commons BY-SA 3.0

Doi: 10.14746/eip.2017.1.4 